\title{
RELATIVISTIC JETS WITH STELLAR WIND ENTRAINMENT.
}

\author{
MARK BOWMAN \\ NRAL, University of Manchester, Jodrell Bank, Nr Macclesfield, Cheshire SK11 9DL, UK.
}

Observations suggest that the flow speeds in extragalactic radio jets are relativistic on parsec scales. This evidence includes rapid variability, brightness temperatures in excess of the inverse Compton limit and even the motion of individual features with apparent superluminal velocities. In contrast, FR-1 flow speeds appear to be non-relativistic on kiloparsec scales (the case is not clear for FR-2 sources); consequently, the possibility of jet deceleration has been explored.

Simulations of steady state jets with both relativistic bulk motion and relativistic equation of state are presented. The effects of entrainment from the stellar winds within the jet are included as source terms in the equations of motion. As cool material is entrained, the flow is forced to decelerate. At the same time dissipation tends to increase the fluid temperature (however, in the jets which were initially hottest [i.e. thermal energy much greater than rest energy] the dissipation is not sufficient to overcome adiabatic cooling). The sub-adiabatic decline in jet pressure and fall in Mach number causes the jet to expand rapidly, as is often observed in real sources.

By expressing the relativistic equation for the evolution of particle number density in a dimensionless form, a parameter can be obtained that expresses the relative importance of entrainment on the jet dynamics. This is:

$$
\kappa=\frac{q z_{c} k T}{P m^{*} \Gamma_{L} c}=\frac{q z_{c}}{\rho \Gamma_{L} c},
$$

where $q$ is the level of entrainment, $z_{c}$ is the core radius of the host galaxy, $k$ is Boltzman's constant, $T$ is the fluid temperature, $P$ is the jet proper pressure, $m^{*}$ is the average particle rest mass, $\Gamma_{L}$ is the Lorentz factor of the flow, $\rho$ is the rest mass density and $c$ is the speed of light. Clearly, initially hot, light jets are more effectively decelerated than equivalent cooler, denser flows. If all jets are assumed to be formed in similiar environments and therefore have similiar escape velocities from the AGN, then the likely deceleration of FR-1 sources to non-relativistic speeds, is indicated to be due to a difference in initial fluid temperature, between these and FR-2 sources. Alternatively, if the two classes of object have initially similiar temperatures then FR-2 sources must have higher initial Lorentz factors. Succintly, the second form of equation (1) indicates that deceleration increases as the jet 'flux' decreases. The effects of stellar wind entrainment on jet opening angles and temperature evolution will be discussed elsewhere.

\section{Acknowledgment}

I would like to express my gratitude to Sergey Komissarov and Patrick Leahy for their useful input on this work. I acknowledge the support of the SERC and the NRAL.

T. J.-L. Courvoisier and A. Blecha: Multi-Wavelength Continuum Emission of AGN, 421.

(C) 1994 IAU. Printed in the Netherlands. 